Communications in Physics, Vol. 25, No. 3 (2015), pp. 247-255

DOI:10.15625/0868-3166/25/3/6147

\title{
FROM MICROSCOPIC GRAVITATIONAL WAVES TO THE QUANTUM INDETERMINISM
}

\author{
VO VAN THUAN \\ Vietnam Atomic Energy Institute (VINATOM) \\ and \\ NEPIO Office, 59 Ly Thuong Kiet street, Hoan Kiem district, Hanoi, Vietnam \\ E-mail: vvthuan@vinatom.gov.vn \\ Received 05 May 2015 \\ Accepted for publication 11 September 2015
}

\begin{abstract}
Based on an extended space-time symmetry a new attempt to search for links between general relativity and quantum mechanics is proposed. A simplified cylindrical model of gravitational geometrical dynamics leads to a microscopic geodesic description of strongly curved extradimensional space-time which implies a duality between an emission law of microscopic gravitational waves and the quantum mechanical equations of free elementary particles. Consequently, the Heisenberg indeterminism would originate from the time-space curvatures.
\end{abstract}

Keywords: time-space symmetry, microscopic gravitational waves, quantum indeterminism.

\section{INTRODUCTION}

A fundamental problem of physics is the consistency of quantum mechanics with general relativity. Kaluza and Klein [1,2] were pioneers to propose a space-like extradimension (ED) which is to compact in a micro circle in a relation to general relativity. Klein and Fock $[2,3]$ also found a formalism that the equation of motion of a massive particle in 4D space-time can be obtained by reducing the EDs of a massless particle in a higher dimensional time-space. For the semi-classical approach to quantum mechanics introduced by de Broglie and Bohm $[4,5]$ the hidden parameters are somehow reminiscent of EDs. Later on, the evidence for violation of Bell inequalities [6,7] abandoned the models with local hidden parameters, however, leaving the door open to non-local hidden variables. In the wake of high dimensional superstring models, another trend has been developed following the Kaluza-Klein geometrical dynamics, of which most applied space-like EDs, while few others considered time-like ones. There are two main approaches with time-like EDs: membrane models in the Anti-de-Sitter geometry (AdS), such as [8,9]and induced matter models $[10,11]$. In particular, Maldacena [8] found a duality between AdS and conformal fields as AdS/CFT formalism. Randall and Sundrum [9] applied an infinite 5D AdS model for a hierarchy solution. For the induced matter approach, Wesson [10] has proposed a spacetime-matter model describing proper mass as a time-like ED. A geometrical dynamic model for 
elementary particles was proposed by Koch $[12,13]$ with a time-like ED which offered a method for derivation of Klein-Gordon equation. Our preliminary study [14] following the induced-matter approach was based on the space-time symmetry in which the Klein-Fock reduction formalism was used. In the present study we do a new attempt in a more direct way to prove that the quantum wave equations in $4 \mathrm{D}$ space-time can be identical to a general relativistic geodesic description of curved extradimensional time-space.

\section{SYMMETRY WITH TIME-LIKE EXTRADIMENSIONS}

Considering two orthogonal subspaces, 3D-time and 3D-space, we construct an ideal 6D flat extended symmetrical time-space $\left\{t_{1}, t_{2}, t_{3} \mid x_{1}, x_{2}, x_{3}\right\}$ :

$$
d S^{2}=d \vec{k}^{2}-d \vec{l}^{2}=d t_{k}^{2}-d x_{l}^{2} .
$$

Our further investigation bases on the time-space symmetrical "lightcone" embedded in the 6D flat time-space (1):

$$
d \vec{k}^{2}=d \vec{l}^{2} \rightarrow d t_{k}^{2}=d x_{l}^{2}
$$

where $k, l=1 \div 3$ are summation indexes that: $d \vec{k}^{2}=\sum d t_{k}^{2}$ and $d \vec{l}^{2}=\sum d x_{l}^{2}$. Hereafter, natural units $(\hbar=c=1)$ are used generally unless it needs an explicit quantum dimension. Any differentials-displacements of $d t_{k}$ and $d x_{l}$ correspondingly in 3D-time and in 3D-space can be independent from each other. They are naturally considered as the most primitive sources of physical potential energy in the symmetrical time-space. The time-like source can form a special global vacuum potential in 3D-time equivalent to the original tachyonic Higgs potential. Another half of the primitive source in 3D-space would contribute to the global cosmological constant $\Lambda$. We assume a postulate that the equality (2) between the squared linear time-like and space-like intervals $d \vec{k}^{2}=d \vec{l}^{2}$ is to accept as a fundamental time-space symmetry not only for Euclidean geometry, but also to be conserved for the linear translational elements of more generalized space-time geometries and in their transformation from a higher dimensional geometry to lower one. This assumption denoted as a principal conservation of linear translation (CLT) bases on our experience of the firm Lorentz invariance as well as of the homogeneity and isotropy of 4D Minkowski space-time.

Now let us consider among differentials (2) those displacements for which a time-like displacement correlates with a space-like one by a harmonic function $f\left(t_{k}, x_{l}\right)$. In the flat 6D timespace we introduce the following $6 \mathrm{D}$ isotropic plane wave equation:

$$
\frac{\partial^{2} f}{\partial t_{k}^{2}}=\frac{\partial^{2} f}{\partial x_{l}^{2}},
$$

where $t_{k}$ and $x_{l}$ remain Descartes coordinates, describing transmission of plane waves $f\left(t_{k}, x_{l}\right)$ in Euclidean time-space. The 6D wave transmission (3) can serve as a primitive energy-momentum formation of physical objects, symmetrical in 3D-time and 3D-space. Most of the primary form of energy in 3D-time probably is almost unobservable from our 3D-space and would be a kind of dark energy. Zeldovich in [15] applied a special global cosmological vacuum to a $\Lambda$-model for elementary particles. Qualitatively, following [15], we would propose a scenario of formation of a single direction of time evolution of microscopic substances, using the time-like vacuum potential. Namely, the global potential in 3D-time is able to generate strong quantum fluctuations 
in space-time. In particular, there are individual fluctuations being able to fix a time-like circular polarization in 3D-time equivalent to breaking of space-time symmetry and similar to the Higgs mechanism to induce the proper mass to a kind of identical elementary particles. The plane wave (3) acquires a time-like circular polarization namely along the $t_{3}$ axis, keeping strictly an arrow evolution from the past to the future and being constrained by a time-like cylindrical condition. Polar coordinates $\left\{\psi\left(t_{0}\right), \varphi\left(t_{0}\right), t_{3}\right\}$ are used for the 3D-time instead of linear coordinates $\left\{t_{k}\right\}$ where vector $d t_{0}$ is evolving along a circle located in a plane orthogonal to the longitudinal vector $d t_{3}$. The squared differential along the time curve reads:

$$
d t^{2}=d \psi\left(t_{0}\right)^{2}+\psi^{2} d \varphi\left(t_{0}\right)^{2}+d t_{3}^{2}=d s^{2}+d t_{3}^{2}
$$

There $d s$ is fixed as the Lorentz invariant interval characterizing the curvature term of time $t$. In cylindrical geometry the only linear term of time is $d t_{3}$ in (4) getting identical to the time-like interval $d \vec{k}$ in (2), in according to the CLT principle. The direction of vector $d t_{0}$ serves a label attached to a particle or an anti-particle. Vectors $d t_{3}$ and $d t_{0}$ form a local basis for their vector summation $d t$ such as $\Omega d t=\Omega_{0} d t_{0}+\Omega_{3} d t_{3}$, where $\Omega, \Omega_{0}$ and $\Omega_{3}$ are linear scale factorization parameters. Therefore, the local time-like geodesic $d t$ in $3 \mathrm{D}$-time plays a role of a real time duration in our 3D-space.

Linear translation of a material point in 3D-space is taken along a linear vector $d \vec{l}$ defined along the same direction of the linear vector $d \vec{l}$ in (2). Simultaneously, the material point can also rotate around an arbitrary 3D-spatial direction characterized by a spin $\vec{s}$. Originally, vector $\vec{s}$ in 3D-space is described by a spherical system attached to the material point $\left\{\psi\left(x_{n}\right), \theta\left(x_{n}\right), \varphi\left(x_{n}\right)\right\}$, where $\psi$ is the deviation variable extended in 3D-space, $\varphi$ is the azimuth around the spherical axis $\vec{l}$, while $\theta$ as the zenith between $\vec{l}$ and $\vec{\psi}$ defines its right-additive as the angle between $\vec{s}$ and $\vec{l}$. For conserving Lorentz invariance, we are restricted to consider the projection $s_{n}$ of $\vec{s}$ on axis $x_{l}$ which defines a local rotation in a plane $P_{n}$ orthogonal to vector $d x_{l}$ and the local proper coordinate $x_{n} \subset P_{n}$ now plays a role of a new affine parameter in 3D-space. The squared differential along the spatial curve reads:

$$
d \lambda^{2}=d \psi\left(x_{n}\right)^{2}+\psi\left(x_{n}\right)^{2}\left[d \theta^{2}+\sin ^{2} \theta d \varphi\left(x_{n}\right)^{2}\right]+d x_{l}^{2}=d \sigma^{2}+d l^{2},
$$

where $d \sigma^{2}=d \sigma_{e v}^{2}+d \sigma_{o d}^{2}$ is a formal local interval in 3D-space; $d \sigma_{e v}$ is the P-even component related to rotation with the spatial symmetry and $d \sigma_{o d}$ is the P-odd component implying a contribution of parity nonconservative (PNC) rotation. The affine coordinate $x_{n}$ is hidden under this local interval $d \sigma$. In general, the P-even term may contain a non-Lorentz invariant component of longitudinal fluctuations, however, the latter is compensated as shown below that does not contribute explicitly to our consideration.

In the extended time-space, $\psi=\psi\left(t_{0}, t_{3}, x_{n}, x_{l}\right)$ and $\varphi=\Omega_{0} t_{0}+\Omega_{3} t_{3}-k_{n} x_{n}-k_{l} x_{l}=\Omega t-$ $k_{j} x_{j}$; physical meaning of $\Omega, \Omega_{0}$ and $\Omega_{3}$ is defined as of time-like rotational velocities, while $k_{j}, k_{n}$ and $k_{l}$ are spatial wave factors. This procedure implies that two independent EDs $\psi$ and $\varphi$ turn into the dynamical parameters depending on other 4D space-time dimensions. Being general spatial coordinates $\left\{x_{j}\right\}=\{x, y, z\}$ now form the new basis of 3D-space, describing both linear translation and rotation. For more explicit definition we put axis $\vec{l} / / d x_{3}$, then $d x=d x_{1}$ and $d y=d x_{2}$ are linear, while $d z^{2}=d \sigma^{2}+d x_{3}^{2}$ makes $d z$ curved. In according to CLT principle, during transformation from 6D time-space to 4D space-time: $f\left(t_{k}, x_{l}\right) \rightarrow \psi_{0}\left(t_{3}, x_{l}\right)=\psi e^{i \varphi}$; it needs to add a Lorentz-like 
condition in 4D space-time for compensation of longitudinal fluctuations, such as:

$$
\left(\frac{\partial \varphi}{\partial t_{3}}\right)^{2}=\left(\frac{\partial \varphi}{\partial x_{l}}\right)^{2}
$$

Then condition (6) allows to conserve the plane wave equation (3) in 4D space-time as well:

$$
\frac{\partial^{2} \psi}{\partial t_{3}^{2}}=\frac{\partial^{2} \psi}{\partial x_{l}^{2}} .
$$

The CLT principle and the definition of basis $\left\{x_{j}\right\}$ in 3D-space allow to obtain $d t_{3}{ }^{2}=d l^{2}$. The 6D "lightcone" geometry (2) being generalized with curvature is turning into a new quadratic form:

$$
d t^{2}-d s^{2}=d t_{3}^{2}=d l^{2}=d \lambda^{2}-d \sigma^{2} .
$$

Accordingly, the 6D time-space representation is transformed to a 4D space-time geometry of a spinning particle describing both translation and rotation:

$$
d \Sigma^{2}=d s^{2}-d \sigma^{2}=d t^{2}-d \lambda^{2}
$$

where $d \Sigma$ is a total 4D interval which is to be fixed as a Lorentz invariant only after a transformation from the 6D time-space to the 4D space-time. The more generalized quadratic form (9) includes, as its partial linearization case, the traditional quadratic form of 4D Minkowski spacetime describing the linear translation:

$$
d s^{2}-d \sigma_{o d}^{2} \approx d s^{2}=d t^{2}-d l^{2}
$$

There $d \sigma_{o d}$ is too small as originated from PNC effect and usually may be ignored. Equation (10) proves the consistency of geometry (9) with special relativity.

\section{GEODESIC DEVIATION FOR GRAVITATIONAL WAVE}

For formulation of the equation of motion in according to transformation from $6 \mathrm{D}$ timespace to 4D space-time we consider a geodesic acceleration equation of deviation $\psi$. The 3D local affine parameters in this equation are the transverse evolving time $t_{0}$ (proper time) and the transverse rotational space-like variables $x_{n}$ (proper spinning). In according to time-space symmetry (2) we postulate that any deviation from the linear translation in 3D-time is to compensate by a deviation in 3D-space, i.e. a balance established between two extended differentials: $D u\left(t_{0}\right)=D u\left(x_{n}\right)$; where the velocity $u(s)=\frac{\partial \psi}{\partial s}$. The non-zero validity of $D u(s)$ means a pumping of $\mathrm{P}$ - or/and T-violations between two 3D subspaces. However, P-violation and T-violation (as well as CP-violation) are weak or superweak, that it is often ignored and makes the 3D local geodesic acceleration conditions in the two subspaces almost absolute and independent. Therefore, the symmetrical equation of geodesic acceleration of deviation $\psi$ in both 3D-time and 3D-space reads:

$$
\frac{\partial^{2} \psi}{\partial t_{0}^{2}}+\Gamma_{\alpha \beta}^{\psi}\left(\frac{\partial t_{\alpha}}{\partial t_{0}}\right)\left(\frac{\partial t_{\beta}}{\partial t_{0}}\right)=\frac{\partial^{2} \psi}{\partial x_{n}^{2}}+\Gamma_{\gamma \sigma}^{\psi}\left(\frac{\partial x_{\gamma}}{\partial x_{n}}\right)\left(\frac{\partial x_{\sigma}}{\partial x_{n}}\right)=0
$$

where $t_{\alpha}, t_{\beta} \in\left\{\psi\left(t_{0}\right), \varphi\left(t_{0}\right), t_{3}\right\}$ and $x_{\gamma}, x_{\sigma} \in\left\{\psi\left(x_{n}\right), \varphi\left(x_{n}\right), x_{l}\right\} ; l, n=1 \div 3$. In both sides of (11) among the related Christoffel symbols except $\Gamma_{\varphi\left(t_{0}\right) \varphi\left(t_{0}\right)}^{\psi}=-\psi$ and $\Gamma_{\varphi\left(x_{n}\right) \varphi\left(x_{n}\right)}^{\psi}=-\psi \cdot \sin ^{2} \theta$, all other terms are vanished or ignored due to orthogonality of $d t_{\alpha}$ (or $d t_{\beta}$ ) to $d t_{0}$ and of $d x_{\gamma}$ (or $d x_{\sigma}$ ) to $d x_{n}$. Applying CLT principle, the differential equation of linear elements (7) can be now added 
to (11) for fully describing the geodesic acceleration of deviation $\psi$ including rotation as well as linear translation:

$$
\frac{\partial^{2} \psi}{\partial t_{0}^{2}}-\psi\left(\frac{\partial \varphi}{\partial t_{0}}\right)^{2}+\frac{\partial^{2} \psi}{\partial t_{3}^{2}}=\frac{\partial^{2} \psi}{\partial x_{n}^{2}}-\psi \sin ^{2} \theta\left(\frac{\partial \varphi}{\partial x_{n}}\right)^{2}+\frac{\partial^{2} \psi}{\partial x_{l}^{2}} .
$$

As in (12) differentials $d t_{3}$ and $d t_{0}$, as well as corresponding covariant derivatives are locally orthogonal to each other, that we can group their second derivatives together as:

$$
\frac{\partial^{2} \psi}{\partial t_{0}+2}+\frac{\partial^{2} \psi}{\partial t_{3}^{2}}=\frac{\partial^{2} \psi}{\partial t^{2}}
$$

where $t_{0}{ }^{+}$means an evolution toward the future. Similarly, due to a local orthogonality, for differentials $d x_{l}$ and $d x_{n}$, the second derivatives in 3D-space are also combined as:

$$
\frac{\partial^{2} \psi}{\partial x_{n}^{2}}+\frac{\partial^{2} \psi}{\partial x_{l}^{2}}=\frac{\partial^{2} \psi}{\partial x_{j}^{2}}
$$

In the result, two operations: i/ defining $\psi$ as a deviation parameter and ii/ the unification (13) of two orthogonal time axes into the ordinary time $t$, almost hide the proper time $t_{0}$ and, simultaneously, reduce the 6D manifold into a 4D space-time. In principle, the vacuum circular polarization can separate time-like geodesic acceleration (as well as space-like one) in two opposite directions of evolution (or rotation). Before a separation of polarization there are symmetrical contributions of forward and backward evolutions in 3D-time and there is a symmetry between left-handed and right-handed contributions in 3D-space. However, due to transformation into 4D space-time and fixing the polarization, this symmetry is to be broken. For running the mechanism, qualitatively assuming that a Higgs-like tachyonic potential $V_{H}(\phi)=\lambda^{2}\left[\phi^{2}-\phi_{0}^{2}\right]^{2}$ induces a time-like centripetal force in 3D-time, where $\phi_{0}$ is the global vacuum field and $\lambda$ is the interaction constant. A centrifugal interaction potential in 3D-space is equivalent to a tachyonic centripetal in 3D-time and vise-versa which would convert to each other by spontaneous breaking of symmetry as: $V_{H}(\phi) \Rightarrow V_{H}(\chi)$ replacing $\phi=\chi+\phi_{0}$. This procedure is accompanied by transformations (13) and (14), simultaneously. Applying a dimensional analysis in natural units: $[$ length $]=[$ time $]=[\text { mass }]^{-1}$, we estimate the major term of acceleration of the time-like deviation $\psi$ in (12) as following:

$$
-\left(\frac{\partial \varphi}{\partial t_{0}}\right)^{2} \psi=\frac{V_{H}(\phi)}{\psi^{2}} \psi \Rightarrow-\left(\frac{\partial \varphi}{\partial t_{0}{ }^{+}}\right)^{2} \psi=\frac{V_{H}(\chi)}{\psi^{2}} \psi=C_{1} m_{H}{ }^{2} \frac{\chi^{2}}{\psi^{2}}\left(1+\frac{\chi}{2 \phi_{0}}\right)^{2} \psi .
$$

The arrow means the breaking symmetry which is equivalent to selecting a single evolution toward the future accompanied by transformation from $6 \mathrm{D}$ time-space to $4 \mathrm{D}$ space-time. We propose the calibration factor of dimension $\left[C_{1}\right]=c^{4} \hbar^{-2}$; a square ratio of time-like radii can be used for a qualitative estimation: $\frac{\chi^{2}}{\psi^{2}} \approx \frac{\left\langle\chi\left|r_{H}^{2}\right| \chi\right\rangle}{\left\langle\psi\left|r_{e}^{2}\right| \psi\right\rangle}=\left(\frac{m_{0}}{m_{H}}\right)^{2}$; where $\left\langle r_{H}^{2}\right\rangle$ and $\left\langle r_{e}^{2}\right\rangle$ are square averaged classical radii of Higgs boson and the interacting elementary particle $e$, respectively. In the result, (15) determines the major term of particle mass in the first order of approximation with: $-\left(\frac{\partial \varphi}{\partial t_{0}+}\right)^{2} \psi \approx$ $m_{0}^{2} \psi$.

The proper mass contains also space-like contribution corresponding to spinning in 3Dspace which consists of P-even and P-odd terms. The squared P-even contribution is $m_{s}^{2} \sim$ 
$\left(k_{n} \cdot \vec{s}\right)_{\text {even }}^{2}$, where $\vec{s}$ is the intrinsic spin of elementary particle and its normally oriented projection $s_{l}$ relative to the rotational plane $P_{n}$ can be both, left or right direction. In principle, electromagnetic or nuclear forces conserving the P-symmetry in 3D-space can be an inducing mechanism of the P-even contribution. Generally, without special polarization tool, the P-even contribution is to be hidden under the local geodesic acceleration condition in 3D-space:

$$
\frac{\partial^{2} \psi}{\partial x_{n}^{2}}-\psi \sin ^{2} \theta\left(\frac{\partial \varphi}{\partial x_{n}}\right)^{2} \approx \frac{\partial^{2} \psi}{\partial x_{n}^{2}}-\psi\left(k_{n} . s_{l}\right)_{\text {even }}^{2}=0
$$

The P-even mass contribution of the spinning term $m_{s}$ can be revealed in special polarization interactions. Instead of that, the P-odd contribution is a global proper polarization effect in term of the left-handed helicity, being observed universally in the weak interaction. We make a qualitative assumption that the breaking of symmetry in P-odd term may be caused by a vacuum potential of the global cosmological constant $\Lambda$ in $3 \mathrm{D}$ space:

$$
\left(\frac{\partial \varphi}{\partial x_{n}}\right)_{o d d}^{2} \psi \Rightarrow\left(\frac{\partial \varphi}{\partial x_{n}^{L}}\right)^{2} \psi=-\hbar^{-2} \omega^{2} s_{L}^{2} \psi
$$

The arrow again means the breaking symmetry by fixing a given helicity; $s_{L}$ is a normal spin projection to the plane $P_{n}$ equivalent to the left-handed helicity. The mass scale factor $\omega$ of P-odd contribution can be estimated from electroweak interference of leptons as $\omega \sim \alpha . G_{F} m_{0}^{3}$; i.e. proportional to the fine structure constant $\alpha$ and Fermi constant $G_{F}$.

Finally, from (12) we obtain the 4D space-time geodesic equation as follows:

$$
-\frac{\partial^{2} \psi}{\partial t^{2}}+\frac{\partial^{2} \psi}{\partial x_{j}^{2}}=-\left[\left(\frac{\partial \varphi}{\partial t_{0}{ }^{+}}\right)^{2}-\left(k_{n} . s_{l}\right)_{\text {even }}^{2}-\left(\frac{\partial \varphi}{\partial x_{n}^{L}}\right)^{2}\right] \psi=-\delta_{M}^{2} \psi .
$$

This nonhomogeneous squared differential equation is a transmission law of the function $\psi$ which characterizes a time-like curvature with a space-like adjustment. Therefore, Equation (18) defines the emission of microscopic gravitational waves from gravitational sources $\delta_{M}^{2} \psi$. At variance with the traditional macroscopic gravitational waves with a very weak curvature carried by graviton, Equation (18) contains a major strong time-like curvature which is inversely proportional to the microscopic wave function $\psi$ carried by different quanta than graviton. Moreover, because the gravitational sources are globally extended everywhere in 6D time-space, the interaction potential is to attach to the moving material point, described by the geodesic equation (18). In the result, the speed of translational transmission of the wave phase is faster than the speed of light, which seems to ban observation. Nevertheless, the energy-matter following Equation (18) is to transmit with an observable subluminal speed. Indeed, going on to rescale (18) with the Planck constant we can find that the microscopic gravitational waves are identical to the well-known and well-observable quantum waves.

\section{QUANTUM EQUATIONS AND INDETERMINISM}

For formulation of quantum mechanical equations from a classical geodesic description, we adopt the quantum dynamic operators, such as: $\frac{\partial}{\partial t} \rightarrow i . \hbar \frac{\partial}{\partial t}=\widehat{E}$ and $\frac{\partial}{\partial x_{j}} \rightarrow-i . \hbar \frac{\partial}{\partial x_{j}}=\widehat{p}_{j}$. For the particle at rest, when $t \rightarrow t_{0}$ and $x_{j} \rightarrow x_{n}$, the operators are getting generators of proper masses: $i . \hbar \frac{\partial}{\partial t_{0}}=\widehat{E_{0}}=\widehat{m_{0}}$ and $-i . \hbar \frac{\partial}{\partial x_{n}}=\widehat{p_{n}}=\widehat{\delta m}$. This traditional procedure of quantum mechanics 
would be interpreted as a conversion between time and space which serves a mean for 4D spacetime macroscopic observation of the microscopic gravitational waves transmitted in according to (18). Consequently, Equation (18) leads to the basic quantum mechanical equation of motion:

$$
-\hbar^{2} \frac{\partial^{2} \psi}{\partial t^{2}}+\hbar^{2} \frac{\partial^{2} \psi}{\partial x_{j}^{2}}-m^{2} \psi=0
$$

where the square mass term $m$ consists of the following components: $m^{2}=m_{0}^{2}-\delta m^{2}=m_{0}^{2}-m_{s}^{2}-$ $m_{L}^{2}$. In momentum representation Equation (18) reads:

$$
E^{2} \psi_{p}-\vec{p}^{2} \psi_{p}-m^{2} \psi_{p}=0 .
$$

Equation (20) describes subluminal motion of an elementary particle with energy $E$ and momentum $\vec{p}$. In comparison with the traditional expression of the rest mass, the present one includes an additional correction $\delta m$ associated with the contribution of the intrinsic spin in 3Dspace. The P-even contribution $m_{s}$ linked with an external curvature of spinning in 3D-space can be compensated in according to the condition (16) when only the linear translation along $x_{l}$ axis is taken in account for a laboratory frame observation. However, due to P-odd effect being observable in the weak interaction, the geodesic deviation of the material point by its spinning still induces a small non-zero mass scale factor $|\omega|=m_{L} \ll m_{s}$ which proves a tiny internal curvature of our realistic 3D-space. In general, Equation (19) is reminiscent of Proca equation of vector boson or the squared Dirac equation of lepton [14]. In case of a scalar field or when there is no polarization analysis, $m \rightarrow m_{0}$, Equation (19) turns to the traditional Klein-Gordon-Fock equation in the linear 3D-space (with $\left\{x_{j}\right\} \rightarrow\left\{x_{l}\right\}$ ):

$$
-\hbar^{2} \frac{\partial^{2} \psi}{\partial t^{2}}+\hbar^{2} \frac{\partial^{2} \psi}{\partial x_{l}^{2}}-m_{0}^{2} \psi=0
$$

It is to mention that the local condition of geodesic deviation (16) in 3D-space leads to:

$$
\left(\frac{\partial S}{\partial x_{n}}\right)^{2}=\left(\hbar \cdot \sin \theta \frac{\partial \varphi}{\partial x_{n}}\right)^{2}=\frac{\hbar^{2}}{\psi} \frac{\partial^{2} \psi}{\partial x_{n}^{2}}=-2 m Q_{B}
$$

which is proportional to Bohm quantum potential $Q_{B}$ in [5].

The existence of the spin term in (19) is reminiscent of the Zitterbewegung of a free spinning electron (Schrodinger ZBW) [16]. In fact, when we describe in a laboratory frame a linear translation of a free particle by Equation (19), the ZBW term is made almost hidden by the geodesic acceleration condition (16) except a tiny P-odd term which is usually hard to observe. This implies a reason why ZBW is not observable experimentally without special measure of polarization or interference.

In according to the condition of geodesic deviation acceleration restricted locally in 3Dtime, from the left side of (11) it follows:

$$
d E_{0} \cdot d t_{0}=\psi^{-1} d\left(i . \hbar \frac{\partial \psi}{\partial t_{0}}\right) \cdot d t_{0}=i . \hbar \cdot d \varphi^{2} .
$$

Based on (23) one can derive the time-energy inequality:

$$
|\Delta E| .|\Delta t| \geq\left|\Delta E_{0}\right| \cdot\left|\Delta t_{0}\right|>\left|d E_{0}\right| \cdot\left|d t_{0}\right|=|i . \hbar| . d \varphi^{2} \geq \Delta \varphi_{\min }^{2} \hbar \geq 0 .
$$


Similarly, the right side of (11) or the local condition of geodesic deviation (16) in 3Dspace leads to the relation:

$$
d p_{n} \cdot d x_{n}=\psi^{-1} d\left(-i . \hbar \frac{\partial \psi}{\partial x_{n}}\right) \cdot d x_{n}=-i . \hbar \cdot \sin ^{2} \theta d \varphi^{2} .
$$

One can now derive the space-momentum inequality:

$$
|\Delta p| \cdot|\Delta x| \geq\left|\Delta p_{n}\right| \cdot\left|\Delta x_{n}\right|>\left|d p_{n}\right| \cdot\left|d x_{n}\right|=|i . \hbar| \cdot \sin ^{2} \theta d \varphi^{2} \geq \Delta \varphi_{\min }^{2} \hbar \geq 0 .
$$

Inequalities (24) and (26) can turn to equalities to zero only for the flat time-space of Euclidean geometry. For a non-zero curvature, it is proposed for (26) an additional condition of $\sin ^{2} \theta=1$, i.e. 3D-space quantization $\theta=(n+1 / 2) \pi$, equivalent to the cylindrical condition, and for both equations adopting $\Delta \varphi_{\text {min }}=\sigma(\langle\varphi\rangle)=\sqrt{2 \pi}$, where due to a statistical observability of the quantum indeterminism, $\sigma$ is a standard deviation of the mean value $\langle\varphi\rangle=2 \pi$ of an appropriate statistical distribution such as Poisson or Gaussian ones. The statistical feature possibly relates to the fact that in according to an anthropic principle, human observation of an individual elementary particle (e.g. an electron) is based on collective effects of interaction between human body and experimental apparatus with this microscopic particle. In practice, even the macroscopic observation system is involved in the same energy-momentum level as of electrons, however, it is only an averaged collective state. Recalling that in [14] we derived the continuity equation of "a single particle" which in combination with the geodesic equation (18) qualitatively allows to understand the physical reality of an individual particle in consistency with the quantum statistical interpretation and the context of the wave-particle duality observed in 4D space-time. In the result, we obtain the Heisenberg inequalities. This proves a direct link between time-space curvature of general relativity and Heisenberg inequalities in quantum mechanics.

\section{CONCLUSIONS}

Based on a simplified model of time-space symmetry, we have shown that the extended 3D-time seems not to be a fictive subspace, but the existence of time-like EDs is revealed in terms of the quantum wave function $\psi$ and the proper time $t_{0}$ (under the time-like rotational parameter $\varphi\left(t_{0}\right)$ ). Being hidden under curvature of the microscopic space-time, those two "transverse" timelike EDs contribute to a full time-space evolution of microscopic elementary particles. A duality was found that the second order differential equations in quantum mechanics, including Proca, squared Dirac and Klein-Gordon-Fock equations, are indeed the emission law of a specific kind of microscopic gravitational waves which carry a strong time-like curvature in a weakly curved 3D-space.

The local 3D geodesic acceleration conditions of deviation $\psi$ shed light on the origin of quantum phenomena such as: i/ Bohm quantum potential, ii/ Schrodinger ZBW of a spinning electron and iii/ Heisenberg inequalities. In particular, triumph of Heisenberg indeterminism serves a strong evidence of internal curvatures of our realistic 4D space-time.

Those observations imply a necessity of reformation of our basic concepts, such as the flatness of 4D space-time and the locality of the dynamical interactions in 4D stage. It is possible to consider quantum mechanics as an effective 4D space-time holography which serves for restoration of the physical world on an extended lightcone surface of 6D symmetrical time-space. 
Finally, the conclusions done here would serve positive arguments for a deep consistency between general relativity and quantum mechanics.

\section{ACKNOWLEDGMENT}

The author is grateful to Nguyen Ai Viet and Nguyen Anh Ky (Hanoi Institute of Physics) for their useful discussion. A hearty thanks is extended to N. B. Nguyen (Hanoi Thang Long Univ.) and M. L. Le-Vos for their technical assistance and precious encouragement.

\section{REFERENCES}

[1] T. Kaluza, Sitz. Preuss. Akad. Wiss. 33 (1921) 966.

[2] O. Klein, Z. f. Physik 37 (1926) 895.

[3] V. Fock, Z. f. Physik 39 (1926) 226.

[4] L. de Broglie, J. Phys. et Radium 8 (1927) 225.

[5] D. Bohm, Phys. Rev. 85 (1952) 166.

[6] J. S. Bell, Physics 1 (1964) 195.

[7] S. J. Freedman and J. F. Clauser, Phys. Rev. Lett. 28 (1972) 938.

[8] J. Maldacena, Adv. Theor. Math. Phys. 2 (1998) 231.

[9] L. Randall and R. Sundrum, Phys. Rev. Lett. 83 (1999) 4690.

[10] P. S. Wesson, Phys. Lett. B 276 (1992) 299.

[11] S. S. Seahra, P. S. Wesson, Gen. Rel. Grav. 33 (2001) 1731.

[12] B. Koch, arXiv:0801.4635v1[quant-ph]2008.

[13] B. Koch, arXiv:0801.4635v2[quant-ph]2009.

[14] Vo Van Thuan, IJMPA 24 (2009) 3545.

[15] Ya.B. Zeldovich, Usp. Fiz. Nauk 95 (1968) 209.

[16] E. Schrodinger, Sitz. Preuss. Akad. Wiss. Phys.-Math. Kl. 24 (1930) 418 